\title{
INTEGRAL REPRESENTATION OF THE SOLUTIONS TO HEUN'S BICONFLUENT EQUATION
}

\author{
S. BELMEHDI AND J.-P. CHEHAB
}

Received 1 October 2002

First, we trace the genesis of the canonical form of Heun's biconfluent equation. Second, we present a method which allows us to find an integral expression as a solution to our equation, and finally, using the properties of Meijer $G$-functions, we give an integral representation of a fundamental system of solutions to the biconfluent equation.

\section{Preliminaries}

Heun's differential equation and its confluent forms are used to build up new classes of solvable potentials. The Schrödinger equation formed with those potentials can be reduced to Heun's biconfluent differential equation. We list some examples:

(i) radial Schrödinger equation for the harmonic oscillator [15];

(ii) radial Schrödinger equation for the doubly anharmonic oscillator $[4,5,10]$;

(iii) radial Schrödinger equation of a three-dimensional anharmonic oscillator [7, 8, $10]$;

(iv) radial Schrödinger equation of a class of confinement potentials [10, 16].

For other kinds of potentials, see $[11,12]$.

Recently, a very interesting and valuable monography was dedicated to Heun's equations [17]. Arscott [1] conjectures that solutions of Heun's equations are not expressible in terms of definite or contour integrals involving simpler functions. One should mention the work of Sleeman who gave a solution in the form of factorial series, which leads to Barnes-type contour integrals [18]. In the sequel, we will see that it is possible to give integral representations in terms of Mellin's kernel of solutions to biconfluent Heun's equation.

We start with the canonical form of a second-order differential equation with $p(p \geq 2)$ elementary singular points ( $p-1$ finite singularities and the $\infty$ ):

$$
y^{\prime \prime}+\sum_{r=1}^{p-1} \frac{2 / p}{x-a_{r}} y^{\prime}+\frac{\sum_{k=1}^{p-3} A_{k} x^{p-3-k}}{\prod_{r=1}^{p-1}\left(x-a_{r}\right)} y=0 .
$$

Copyright (C) 2004 Hindawi Publishing Corporation

Abstract and Applied Analysis 2004:4 (2004) 295-306

2000 Mathematics Subject Classification: 33C20, 33C90, 33E30, 33E99, 34L99, 35C15, 34B30

URL: http://dx.doi.org/10.1155/S1085337504306019 
Many differential equations which occur in a large variety of problems arising from pure or applied mathematics or mathematical physics, often after appropriate algebraic or transcendental changes of variable, can be derived by the confluences of the singularities from (1.1). The classes of these equations are characterized by the Klein-Böcher-Ince $\operatorname{symbol}\left(\ell, q, r_{1}, r_{2}, \ldots, r_{s}\right)$ with

$$
p=\ell+2 q+\sum_{k=1}^{s}(k+2) r_{k}
$$

where $\ell$ is the number of elementary singular points, $q$ is the number of nonelementary regular singular points, and $r_{k}$ is the number of irregular singular points of kind $k$. For the terminology, see [9].

If we set $p=8$ by means of confluence process and after parametric reduction, we mention hereby some remarkable equations.

(1) Heun's equation $(0,4,0)$. The confluence of $a_{7}, a_{6} \rightarrow 0, a_{5}, a_{4} \rightarrow a, a_{3}, a_{2} \rightarrow 1$, and $a_{1} \rightarrow \infty$ leads to

$$
y^{\prime \prime}(x)+\left(\frac{\alpha}{x}+\frac{\beta}{x-1}+\frac{\gamma}{x-a}\right) y^{\prime}(x)+\frac{\delta \eta(x-\lambda)}{x(x-1)(x-a)} y(x)=0,
$$

where $\alpha, \beta, \gamma, \delta, \lambda$, and $a$ are six independent parameters and $\eta=\alpha+\beta+\gamma-\delta-1$.

(2) Confluent Heun's equation $\left(0,2,1_{2}\right)$. The confluence of $a_{7}, a_{6}, a_{5} \rightarrow \infty, a_{4}, a_{3} \rightarrow 1$, and $a_{2}, a_{1} \rightarrow 0$ leads to

$$
\begin{aligned}
y^{\prime \prime}(x) & +\left(\alpha+\frac{\beta+1}{x}+\frac{\gamma+1}{x-1}\right) y^{\prime}(x) \\
& +\frac{[\delta+(1 / 2)(\alpha+\gamma+2)] x+\eta+\beta / 2+(1 / 2)(\gamma-\alpha)(\beta+1)}{x(x-1)} y(x)=0,
\end{aligned}
$$

with five independent parameters: $\alpha, \beta, \gamma, \delta$, and $\eta$.

(3) Biconfluent of Heun's equation $\left(0,1,1_{4}\right)$. The confluence of $a_{7}, a_{6}, a_{5}, a_{4}, a_{3} \rightarrow \infty$ and $a_{2}, a_{1} \rightarrow 0$ leads to

$$
\begin{aligned}
x y^{\prime \prime}(x) & +\left(1+\alpha-\beta x-2 x^{2}\right) y^{\prime}(x) \\
& +\left[(\gamma-\alpha-2) x-\frac{1}{2}(\delta+\beta+\alpha \beta)\right] y(x)=0,
\end{aligned}
$$

with four independent parameters: $\alpha, \beta, \gamma$, and $\delta$.

(4) Double confluent of Heun's equation $\left(0,0,2_{2}\right)$. The confluence of $a_{7}, a_{6}, a_{5} \rightarrow \infty$ and $a_{4}, a_{3}, a_{2}, a_{1} \rightarrow 0$ leads to

$$
\begin{aligned}
x^{2} y^{\prime \prime}(x) & +\left[1+\alpha\left(x+\frac{1}{x}\right)\right] x y^{\prime}(x) \\
+ & {\left[\left(\beta-\frac{\alpha}{2}\right) \frac{1}{x}+\frac{\delta \alpha^{2}}{2}-\frac{1}{4}+\left(\gamma+\frac{\alpha}{2}\right) x\right] y(x)=0, }
\end{aligned}
$$

with four independent parameters: $\alpha, \beta, \gamma$, and $\delta$. 
(5) Triconfluent of Heun's equation $\left(0,0,1_{6}\right)$. The confluence of $a_{7}, a_{6}, a_{5}, a_{4}, a_{3}, a_{2}$, $a_{1} \rightarrow \infty$ leads to

$$
y^{\prime \prime}(x)+\left(\gamma+3 x^{2}\right) y^{\prime}(x)+[\alpha+(\beta-3) x] y(x)=0,
$$

with three independent parameters: $\alpha, \beta$, and $\gamma$.

\section{The statement of the problem}

Let $\mathscr{L}_{x}$ be a three-term differential operator $[2,3,9]$

$$
\mathscr{L}_{x}=\frac{P_{1}(\theta)}{x}+P_{0}(\theta)+x R_{1}(\theta)
$$

where $P_{1}(\theta), P_{0}(\theta)$, and $R_{1}(\theta)$ are polynomials and $\theta=x(d / d x)$.

We are looking for a solution to

$$
\mathscr{L}_{x}[y]=0
$$

as

$$
y(x)=\int_{C} K(x, t) Z(t) d t
$$

where

$$
K(x, t)=\frac{K_{1}(x t)}{x}+K_{0}(x t)+x L_{1}(x t) .
$$

The path of integration $C$ and the function $Z(t)$ will be defined in the sequel. We respectively introduce an auxiliary kernel and a companion differential operator:

$$
\begin{gathered}
\tilde{K}(x, t)=t \tilde{K}_{1}(x t)+\tilde{K}_{0}(x t)+\frac{\tilde{L}_{1}(x t)}{t}, \\
M_{t}=t \tilde{P}_{1}(\theta)+\tilde{P}_{0}(\theta)+\frac{\tilde{R}_{1}(\theta)}{t} .
\end{gathered}
$$

In this last equation $\theta$ symbolizes the operator $t(d / d t)$. We have the following assumption:

$$
\mathscr{L}_{x}[K(x, t)]=M_{t}[\tilde{K}(x, t)] .
$$

We denote by $\bar{M}_{t}$ and $A(\tilde{K}, Z)$, respectively, the formal adjoint of $\mathcal{M}_{t}$ and the concomitant (a bilinear functional of $\tilde{K}, Z$ and their derivatives).

If

$$
\bar{M}_{t}[Z(t)]=0 \text {, }
$$

and $A(\tilde{K}, Z)\rfloor_{C}=0$, then $(2.3)$ is a solution to (2.2). 
298 Heun's biconfluent equation-integral representation

Setting $\zeta=x t,(2.6)$ may be translated into the following system:

$$
\begin{gathered}
P_{1}(\theta-1)\left[K_{1}(\zeta)\right]=\zeta^{2} \tilde{P}_{1}(\theta+1)\left[\tilde{K}_{1}(\zeta)\right], \\
P_{1}(\theta)\left[K_{0}(\zeta)\right]+P_{0}(\theta-1)\left[K_{1}(\zeta)\right]=\zeta\left\{\tilde{P}_{1}(\theta)\left[\tilde{K}_{0}(\zeta)\right]+\tilde{P}_{0}(\theta+1)\left[\tilde{K}_{1}(\zeta)\right]\right\} \\
P_{1}(\theta+1)\left[L_{1}(\zeta)\right]+P_{0}(\theta)\left[K_{0}(\zeta)\right]+R_{1}(\theta-1)\left[K_{1}(\zeta)\right] \\
=\tilde{P}_{1}(\theta-1)\left[\tilde{L}_{1}(\zeta)\right]+\tilde{P}_{0}(\theta)\left[\tilde{K}_{0}(\zeta)\right]+\tilde{R}_{1}(\theta+1)\left[\tilde{K}_{1}(\zeta)\right], \\
\zeta\left\{P_{0}(\theta+1)\left[L_{1}(\zeta)\right]+R_{1}(\theta)\left[K_{0}(\zeta)\right]\right\}=\tilde{P}_{0}(\theta-1)\left[\tilde{L}_{1}(\zeta)\right]+\tilde{R}_{1}(\theta)\left[\tilde{K}_{0}(\zeta)\right], \\
\zeta^{2} R_{1}(\theta+1)\left[L_{1}(\zeta)\right]=\tilde{R}_{1}(\theta-1)\left[\tilde{L}_{1}(\zeta)\right] .
\end{gathered}
$$

According to the study in $[2,3]$, the previous system may be reduced to

$$
\begin{aligned}
P_{1}(\theta)\left[K_{0}(\zeta)\right] & =\zeta \tilde{P}_{0}(\theta+1)\left[\tilde{K}_{1}(\zeta)\right], \\
P_{0}(\theta)\left[K_{0}(\zeta)\right] & =\tilde{P}_{0}(\theta)\left[\tilde{K}_{0}(\zeta)\right] \\
\zeta R_{1}(\theta)\left[K_{0}(\zeta)\right] & =\tilde{P}_{1}(\theta-1)\left[\tilde{L}_{1}(\zeta)\right] .
\end{aligned}
$$

In the last system, we have three equations for four unknowns. To solve this system, we have to choose two basic equations and an interdependency relation between the components of the auxiliary kernel. Our choice will be guided by the kind of solution we are looking for.

\section{Heun's biconfluent equation}

The canonical form of an equation of class $\left(0,1,1_{4}\right)$ reads (see $\left.[6,14]\right)$

$$
x y^{\prime \prime}(x)+\left(1+\alpha-\beta x-2 x^{2}\right) y^{\prime}(x)+\left\{(\gamma-\alpha-2) x-\frac{1}{2}[\delta+(1+\alpha) \beta]\right\} y(x)=0 .
$$

Using the operator $\theta=x(d / d x)$, we get that

$$
\left\{\frac{1}{x} \theta(\theta+\alpha)-\left(\beta \theta+-\frac{1}{2}[\delta+(1+\alpha) \beta]\right)+x(\gamma-\alpha-2-2 \theta)\right\}[y]=0 .
$$

We set

$$
\begin{aligned}
& P_{1}(\theta)=\theta(\theta+\alpha), \\
& P_{0}(\theta)=-\beta(\theta+a),
\end{aligned}
$$

where $a=(1 / 2)(\delta / \beta+\alpha+1)$, with $\beta \neq 0$, and

$$
R_{1}(\theta)=-2(\theta+b)
$$

where $b=(\alpha-\gamma+2) / 2$. 
According to the scheme described in Section 2, the companion operator reads

$$
M_{t}=\tilde{P}_{0}(\theta)=\theta+1+d,
$$

where $d \in \mathbb{C}$.

To solve the system defined by (2.9), (2.10), and (2.11), we will take the first two equations as basic equations; the interdependency relation is

$$
\tilde{K}_{1}=\lambda \tilde{K}_{0}
$$

with $\lambda \in \mathbb{C}^{*}$. By elimination, we obtain

$$
\left\{\tilde{P}_{0}(\theta-1) P_{1}(\theta)-\lambda \zeta P_{0}(\theta+1) \tilde{P}_{0}(\theta)\right\}\left[K_{0}\right]=0
$$

Taking into account (3.3), (3.4), and (3.5), we have

$$
\{\theta(\theta+\alpha)(\theta+d)+\beta \lambda \zeta(\theta+2+d)(\theta+a)\}\left[K_{0}\right]=0 .
$$

If we take

$$
\lambda=-\frac{1}{\beta},
$$

then $K_{0}$ satisfies

$$
\{\theta(\theta+\alpha)(\theta+d)-\zeta(\theta+2+d)(\theta+a)\}\left[K_{0}\right]=0
$$

which is nothing but a generalized hypergeometric differential equation whose solutions may be expressed as

$$
\begin{aligned}
& K_{0}(\zeta)={ }_{2} F_{2}\left(\begin{array}{c}
2+d, a \\
1+d, 1+\alpha
\end{array} \mid \zeta\right), \\
& K_{0}(\zeta)=\zeta^{-\alpha}{ }_{2} F_{2}\left(\begin{array}{l}
2-\alpha+d, a-\alpha \\
1-\alpha+d, 1-\alpha
\end{array} \mid \zeta\right), \\
& K_{0}(\zeta)=\zeta^{-d}{ }_{2} F_{2}\left(\begin{array}{c}
2, a-d \\
1-d, 1+\alpha-d
\end{array} \mid \zeta\right) .
\end{aligned}
$$

3.1. First integral representation. In this subsection, we will use the kernel given by (3.12). First, we will compute the components of the auxiliary kernel. From (2.10), we have

$$
\tilde{K}_{0}(\zeta)=\left[\tilde{P}_{0}(\theta)\right]^{-1}\left[P_{0}(\theta)\left[K_{0}(\zeta)\right]\right]
$$

If we take into account (3.4), (3.6), and (3.12), then (3.15) becomes

$$
\tilde{K}_{0}(\zeta)=-\beta(\theta+1+d)^{-1}\left[( \theta + a ) \left[{ }_{2} F_{2}\left(\begin{array}{c}
2+d, a \\
1+d, 1+\alpha
\end{array} \mid \zeta\right)\right.\right.
$$


Using the properties of the operator $\theta$, we get that

$$
\tilde{K}_{0}(\zeta)=-\frac{a \beta \zeta}{1+d}\left\{\frac{1}{1+\alpha}{ }_{1} F_{1}\left(\begin{array}{l}
1+a \\
2+\alpha
\end{array} \mid \zeta\right)+\zeta^{-1}{ }_{1} F_{1}\left(\begin{array}{c}
a \\
1+\alpha
\end{array} \mid \zeta\right)\right\}
$$

with $\mathfrak{R}(1+d)>0$.

According to (3.5), (3.6), and (3.12), $\tilde{L}_{1}$ satisfies the following differential equation:

$$
\tilde{L}_{1}(\zeta)=-2 \zeta(\theta+1+d)^{-1}\left[(\theta+b)\left[{ }_{2} F_{2}\left(\begin{array}{c}
2+d, a \\
1+d, 1+\alpha
\end{array} \mid \zeta\right)\right]\right]
$$

The solution to the previous equation is

$$
\tilde{L}_{1}(\zeta)=-\frac{2 \zeta^{2}}{1+d}\left\{\frac{a}{1+\alpha}{ }_{1} F_{1}\left(\begin{array}{c}
1+a \\
1+\alpha
\end{array} \mid \zeta\right)+b \zeta^{-1}{ }_{1} F_{1}\left(\begin{array}{c|c}
a \\
1+\alpha
\end{array} \mid \zeta\right)\right\}
$$

with $\mathfrak{R}(1+d)>0$.

Thus, the auxiliary kernel reads

$$
\begin{aligned}
& \tilde{K}(x, t)=\frac{1}{1+d}\left\{\frac{a x t^{2}}{1+\alpha}\left[1-\frac{2 x+\beta}{t}\right]{ }_{1} F_{1}\left(\begin{array}{l}
1+a \\
2+\alpha
\end{array} \mid x t\right)\right. \\
& \left.+t\left[a-\frac{2 b x+a \beta}{t}\right]{ }_{1} F_{1}\left(\begin{array}{c}
a \\
1+\alpha
\end{array} \mid x t\right)\right\}
\end{aligned}
$$

with $\mathfrak{R}(1+d)>0$.

Using (3.6), the solution to (2.7) takes the form

$$
Z(t)=t^{d}
$$

The concomitant associated with (2.6) is given by

$$
A(\tilde{K}, Z)=t Z(t) \tilde{K}(x, t) .
$$

The conjunction of (3.20), (3.21), and (3.22) leads to

$$
\begin{aligned}
A(\tilde{K}, Z)=\frac{1}{1+d}\{ & \frac{a x t^{3+d}}{1+\alpha}\left[1-\frac{2 x+\beta}{t}\right]{ }_{1} F_{1}\left(\begin{array}{c}
1+a \\
2+\alpha
\end{array} \mid x t\right) \\
& \left.+t^{2+d}\left[a-\frac{2 b x+a \beta}{t}\right]{ }_{1} F_{1}\left(\begin{array}{c}
a \\
1+\alpha
\end{array} \mid x t\right)\right\},
\end{aligned}
$$

with $\mathfrak{R}(1+d)>0$.

Now it is time to seek for a path of integration along which the concomitant will vanish. With this end in view, we need asymptotic expansion of generalized hypergeometric function which is obtainable via $G$-functions. 
Proposition 3.1 [13]. Recall that

$$
{ }_{1} F_{1}\left(\begin{array}{l}
A \\
B
\end{array} \mid u\right)=\frac{\Gamma(B)}{\Gamma(A)} G_{1,2}^{1,1}\left(-u \mid \begin{array}{c}
1-A \\
0,1-B
\end{array}\right)
$$

where $G_{1,2}^{1,1}$ is a Meijer G-function.

If $a_{1} \notin \mathbb{N}, a_{1} \in \mathbb{C}$, and $\delta_{1}-\pi / 2 \leq \operatorname{Arg}(u) \leq \pi / 2+\delta_{2}, \delta_{1}, \delta_{2}>0$, then

$$
G_{1,2}^{1,1}\left(-u \mid \begin{array}{c}
a_{1} \\
b_{1}, b_{2}
\end{array}\right)=u^{a_{1}-1}\left\{\sum_{k=0}^{N-1} M_{k} u^{-k}+O\left(u^{-N}\right)\right\}, \quad|u| \longrightarrow \infty,
$$

with

$$
\begin{gathered}
M_{k}=\frac{\Gamma\left(1+b_{1}-a_{1}\right)}{\Gamma\left(a_{1}-b_{2}\right)} \frac{\left(1+b_{1}-a_{1}, k\right)\left(1+b_{2}-a_{1}, k\right)}{k !}, \\
(\gamma, k)=\frac{\Gamma(\gamma+k)}{\Gamma(\gamma)} .
\end{gathered}
$$

Corollary 3.2. If $A \notin\{1-n, n \in \mathbb{N}\}$, and $\delta_{1}-\pi / 2 \leq \operatorname{Arg}(u) \leq \pi / 2+\delta_{2}, \delta_{1}, \delta_{2}>0$, then

$$
{ }_{1} F_{1}\left(\begin{array}{l}
A \\
B
\end{array} \mid u\right)=u^{-A}\left\{\sum_{k=0}^{N-1} \tilde{M}_{k} u^{-k}+O\left(u^{-N}\right)\right\}, \quad|u| \longrightarrow \infty
$$

where $\tilde{M}_{k}=(\Gamma(B) / \Gamma(A)) M_{k}$.

Finally, we have

$$
\begin{aligned}
A(\tilde{K}, Z)=\frac{t^{2-a+d}}{1+d}\{ & a x^{-a}\left(1-\frac{2 x+\beta}{t}\right)\left[\sum_{k=0}^{N-1} \tilde{M}_{1, k}(x t)^{-k}+O\left((x t)^{-N}\right)\right] \\
& \left.+x^{-a}\left(a-\frac{2 b x+a \beta}{t}\right)\left[\sum_{k=0}^{N-1} \tilde{M}_{2, k}(x t)^{-k}+O\left((x t)^{-N}\right)\right]\right\},
\end{aligned}
$$

with

(i)

$$
\begin{aligned}
& \tilde{M}_{1, k}=\frac{\Gamma(2+\alpha)}{\Gamma(1+\alpha-a)} \frac{(1+a, k)(a-\alpha, k)}{k !}, \\
& \tilde{M}_{2, k}=\frac{\Gamma(1+\alpha)}{\Gamma(1+\alpha-a)} \frac{(a, k)(a-\alpha, k)}{k !},
\end{aligned}
$$

(ii) $a \notin\{1-n, n \in \mathbb{N}\}$ and $\mathfrak{R}(1+d)>0$,

(iii) $\delta_{1}+\pi / 2 \leq \operatorname{Arg}(x t) \leq 3 \pi / 2+\delta_{2}, \delta_{1}, \delta_{2}>0$.

In accordance with what we have already seen, we have the following theorem. 
Theorem 3.3. If $a \notin\{1-n, n \in \mathbb{N}\}, \mathfrak{R}(a-\alpha)>\mathfrak{R}(1+d)>0, \delta_{1}+\pi / 2 \leq \operatorname{Arg}(x t) \leq$ $3 \pi / 2+\delta_{2}, \delta_{1}, \delta_{2}>0$, and $C$ is the path of integration running from $\infty$ along the direction $\operatorname{Arg}(t)$, surrounding the origin and going back to $\infty$ following the same direction, then

$$
y(x)=\int_{C}{ }_{2} F_{2}\left(\begin{array}{c}
2+d, a \\
1+d, 1+\alpha
\end{array} \mid x t\right) t^{d} d t
$$

is a solution of Heun's biconfluent equation.

3.2. The second integral representation. Now we will work with the kernel given by (3.13), that is,

$$
K_{0}(\zeta)=\zeta^{-\alpha}{ }_{2} F_{2}\left(\begin{array}{c}
2-\alpha+d, a-\alpha \\
1-\alpha+d, 1-\alpha
\end{array} \mid \zeta\right)
$$

Using the same technique as above, we get that

$$
\begin{aligned}
\tilde{K}(x, t)=\frac{1}{1-\alpha+d}\{ & \frac{(a-\alpha) x^{1-\alpha} t^{2-\alpha}}{1-\alpha}\left(\frac{1-2 x}{t}-\frac{1}{\beta}\right){ }_{1} F_{1}\left(\begin{array}{c}
1+a-\alpha \\
2-\alpha
\end{array} \mid x t\right) \\
& \left.+x^{-\alpha} t^{1-\alpha}\left(\frac{a-\alpha-2(b-\alpha) x}{t}-\frac{a-\alpha}{\beta}\right){ }_{1} F_{1}\left(\begin{array}{c}
a-\alpha \\
1-\alpha
\end{array} \mid x t\right)\right\},
\end{aligned}
$$

with $\mathfrak{R}(a-\alpha+d)>0$, and the concomitant takes the following expression:

$$
\begin{aligned}
A(\tilde{K}, Z)=\frac{x^{1-\alpha} t^{3-\alpha+d}}{1-\alpha+d}\{ & \frac{a-\alpha}{1-\alpha}\left(\frac{1-2 x}{t}-\frac{1}{\beta}\right){ }_{1} F_{1}\left(\begin{array}{c}
1+a-\alpha \\
2-\alpha
\end{array} \mid x t\right) \\
& \left.+\frac{1}{x t}\left(\frac{a-\alpha-2 x(b-\alpha)}{t}-\frac{a-\alpha}{\beta}\right){ }_{1} F_{1}\left(\begin{array}{c}
a-\alpha \\
1-\alpha
\end{array} \mid x t\right)\right\} .
\end{aligned}
$$

Using the machinery of $G$-functions, we have the following proposition.

Proposition 3.4. If $a-\alpha \notin\{1-n, n \in \mathbb{N}\}, \mathfrak{R}(\alpha)<\mathfrak{R}(1+d)<\mathfrak{R}(a-1), \delta_{1}+\pi / 2 \leq$ $\operatorname{Arg}(x t) \leq 3 \pi / 2+\delta_{2}, \delta_{1}, \delta_{2}>0$, and $C$ is the path of integration running from $\infty$ along the direction $\operatorname{Arg}(t)$, surrounding the origin and going back to $\infty$ following the same direction, then

$$
A(\tilde{K}, Z)\rfloor_{C}=0
$$

Under the hypothesis of the previous proposition, we get the following theorem.

Theorem 3.5. Provided that the hypotheses of Proposition 3.4 are satisfied, then

$$
y(x)=\int_{C}(x t)^{-\alpha}{ }_{2} F_{2}\left(\begin{array}{c}
2-\alpha+d, a-\alpha \\
1-\alpha+d, 1-\alpha
\end{array} \mid x t\right) t^{d} d t
$$

is a solution of Heun's biconfluent equation. 
The conjunction of Theorems 3.3 and 3.5 gives a fundamental system of solutions to Heun's biconfluent equation.

Remarks. A similar study shows that

(i) the kernel $K_{0}(\zeta)=\zeta^{-d}{ }_{2} F_{2}\left(\begin{array}{c}2, a-d \\ 1-d, 1+\alpha-d\end{array} \mid \zeta\right)$ does not lead to a solution;

(ii) the interdependency relations

$$
\tilde{K}_{1}=\gamma \tilde{L}_{1}, \quad \tilde{K}_{0}=\mu \tilde{L}_{1}
$$

do not allow to produce a solution to the biconfluent equation.

4. Case $\beta=0$

The Heun's biconfluent equation reads

$$
x y^{\prime \prime}(x)+\left(1+\alpha-2 x^{2}\right) y^{\prime}(x)+\left\{(\gamma-\alpha-2) x-\frac{\delta}{2}\right\} y(x)=0
$$

or

$$
\left\{\frac{\theta_{x}\left(\theta_{x}+\alpha\right)}{x}-\frac{\delta}{2}-2 x\left(\theta_{x}+\frac{2+\alpha-\gamma}{2}\right)\right\}[y(x)]=0
$$

This situation gives rise to two subcases

4.1. Case $\delta=0$. Heun's biconfluent equation becomes a simple hypergeometric equation, that is,

$$
x y^{\prime \prime}(x)+\left(1+\alpha-2 x^{2}\right) y^{\prime}(x)+(\gamma-\alpha-2) x y(x)=0,
$$

which has

$$
\begin{aligned}
& y_{1}(x)={ }_{1} F_{1}\left(\begin{array}{c}
\frac{2+\alpha-\gamma}{4} \\
1+\frac{\alpha}{2}
\end{array}\right), x^{2} \\
& y_{2}(x)=x^{-\alpha}{ }_{1} F_{1}\left(\begin{array}{c}
\frac{2-\alpha-\gamma}{4} \\
1-\frac{\alpha}{2}
\end{array}\right)
\end{aligned}
$$

as a fundamental system of solutions that admits an integral representation of Mellin's type (see [2]).

4.2. Case $\delta \neq 0$. In this case, (3.8) becomes

$$
\left\{\theta_{\zeta}\left(\theta_{\zeta}+\alpha\right)\left(\theta_{\zeta}+d\right)+\frac{\lambda \delta}{2} \zeta\left(\theta_{\zeta}+2+d\right)\right\}\left[K_{0}(\zeta)\right]=0 .
$$


304 Heun's biconfluent equation-integral representation

Choosing $\lambda=-2 / \delta$, the previous equation takes the following form:

$$
\left\{\theta_{\zeta}\left(\theta_{\zeta}+\alpha\right)\left(\theta_{\zeta}+d\right)-\zeta\left(\theta_{\zeta}+2+d\right)\right\}\left[K_{0}(\zeta)\right]=0
$$

Hence

$$
\begin{aligned}
& K_{0}(\zeta)={ }_{1} F_{2}\left(\begin{array}{c}
2+d \\
1+d, 1+\alpha
\end{array} \mid \zeta\right), \\
& K_{0}(\zeta)=\zeta^{-\alpha}{ }_{1} F_{2}\left(\begin{array}{c}
2-\alpha+d \\
1-\alpha+d, 1-\alpha
\end{array} \mid \zeta\right), \\
& K_{0}(\zeta)=\zeta^{-d}{ }_{1} F_{2}\left(\begin{array}{c}
2 \\
1-d, 1+\alpha-d
\end{array} \mid \zeta\right) .
\end{aligned}
$$

Proceeding as in Section 3, we get the following proposition.

Proposition 4.1. The pairs of auxiliary kernel and the concomitant associated with (2.6) are given by

$$
\begin{aligned}
& K(x, t)=\frac{t}{1+d}\{ {\left[\frac{2-\delta}{2}-(2+\alpha-\gamma) \frac{x}{t}\right]{ }_{0} F_{1}\left({ }_{1+\alpha} \mid x t\right) } \\
&\left.-\frac{2 x}{1+\alpha}{ }_{0} F_{1}(2+\alpha \mid x t)\right\}, \\
& A(\tilde{K}, Z)=\frac{t^{2+d}}{1+d}\left\{\left[\frac{2-\delta}{2}-(2+\alpha-\gamma) \frac{x}{t}\right]{ }_{0} F_{1}\left({ }_{1+\alpha} \mid x t\right)\right. \\
&-\left.\frac{2 x}{1+\alpha}{ }_{0} F_{1}(2+\alpha \mid x t)\right\}, \\
& K(x, t)=\frac{x^{-\alpha} t^{1-\alpha}}{1-\alpha+d}\left\{\left[1-\frac{\delta+2 x(2-\alpha-\gamma)}{t}\right]{ }_{0} F_{1}\left({ }_{1-\alpha} \mid x t\right)\right. \\
&\left.\quad-\frac{2 x^{2}}{1-\alpha}{ }_{0} F_{1}\left({ }_{2-\alpha} \mid x t\right)\right\}, \\
& A(\tilde{K}, Z)=\frac{x^{-\alpha} t^{2+d-\alpha}}{1+d-\alpha}\left\{\left[1-\frac{\delta+2 x(2-\alpha-\gamma)}{t}\right]{ }_{0} F_{1}\left({ }_{1-\alpha} \mid x t\right)\right. \\
&\left.\quad-\frac{2 x^{2}}{1-\alpha}{ }_{0} F_{1}(2-\alpha \mid x t)\right\} .
\end{aligned}
$$

Remark 4.2. The kernel given by (4.9) does not lead to an integral representation of a solution.

Theorem 4.3. If $0<\mathfrak{R}(\alpha)<\mathfrak{R} 1+d<(1 / 4) \mathfrak{R}(2 \alpha-3), \mu \leq \operatorname{Arg}(x t) \leq 2 \pi-\mu, \mu>0$, and $C$ is the path of integration running from $\infty$ along the direction $\operatorname{Arg}(t)$, surrounding the 
origin and going back to $\infty$ following the same direction, then

$$
\begin{aligned}
& y_{1}(x)=\int_{C}{ }_{1} F_{2}\left(\begin{array}{c}
2+d \\
1+d, 1+\alpha
\end{array} \mid x t\right) t^{d} d t \\
& y_{1}(x)=\int_{C}(x t)^{-\alpha}{ }_{1} F_{2}\left(\begin{array}{c}
2-\alpha+d \\
1-\alpha+d, 1-\alpha
\end{array} \mid x t\right) t^{d} d t
\end{aligned}
$$

is a fundamental system of solutions to the Heun's biconfluent equation (4.1).

\section{References}

[1] F. M. Arscott, Heun's equation, Heun's Differential Equation (A. Ronveaux, ed.), Oxford University Press, New York, 1995.

[2] S. Belmehdi, Une méthode des représentations intégrales en cascade des solutions des équations différentielles à coefficients polynomiaux, Thèse, Université Pierre et Marie Curie - Paris VI, Paris, 1983.

[3] S. Belmehdi and P. Maroni, Une généralisation de la méthode de Mellin, Publ. Labo. Anal. Num. Paris VI, 1985, no. 85006 (French).

[4] R. N. Chaudhuri, The Hill determinant: an application to a class of confinement potentials, J. Phys. A 16 (1983), no. 1, 209-211.

[5] R. N. Chaudhuri and B. Mukherjee, On the $\mu x^{2}+\lambda x^{4}+\eta x^{6}$ interaction, J. Phys. A 17 (1984), no. 17, 3327-3334.

[6] A. Decarreau, P. Maroni, and A. Robert, Sur les équations confluentes de l'équation de Heun [On the confluent equations of the Heun equation], Ann. Soc. Sci. Bruxelles Sér. I 92 (1978), no. 3, 151-189 (French).

[7] G. P. Flessas and G. S. Anagnostatos, On the applicability of the Hill determinant and the analytic continued fraction method to anharmonic oscillators, J. Phys. A 15 (1982), no. 10, L537L542.

[8] G. P. Flessas and K. P. Das, On the three-dimensional anharmonic oscillator, Phys. Lett. A 78 (1980), no. 1, 19-21.

[9] E. L. Ince, Ordinary Differential Equations, Dover Publications, New York, 1926.

[10] B. Léauté and G. Marcilhacy, On the Schrödinger equations of rotating harmonic, threedimensional and doubly anharmonic oscillators and a class of confinement potentials in connection with the biconfluent Heun differential equation, J. Phys. A 19 (1986), no. 17, 35273533.

[11] B. Léauté, G. Marcilhacy, R. Pons, and J. Skinazi, On the connection problem for some Schrödinger equations in relation to the biconfluent Heun differential equation, SIAM J. Math. Anal. 21 (1990), no. 3, 793-798.

[12] A. Lemieux and A. K. Bose, Construction de potentiels pour lesquels l'équation de Schrödinger est soluble, Ann. Inst. H. Poincaré Sect. A (N.S.) 10 (1969), no. 3, 259-270 (French).

[13] Y. L. Luke, The Special Functions and Their Approximations, Vol. I, Mathematics in Science and Engineering, vol. 53, Academic Press, New York, 1969.

[14] P. Maroni, Sur la forme bi-confluente de l'équation de Heun, C. R. Acad. Sci. Paris Sér. A-B 264 (1967), A503-A505 (French).

[15] D. Masson, The rotating harmonic oscillator eigenvalue problem. I. Continued fractions and analytic continuation, J. Math. Phys. 24 (1983), no. 8, 2074-2088.

[16] R. Pons and G. Marcilhacy, Contribution à l'étude de l'équation de Schrödinger avec un potentiel de Coulomb et un terme de confinement linéaire [Contribution to the study of the Schrödinger equation with a Coulomb potential and a linear confinement term], Ann. Soc. Sci. Bruxelles Sér. I 102 (1988), no. 1-2, 3-17 (French). 


\section{Heun's biconfluent equation-integral representation}

[17] A. Ronveaux (ed.), Heun's Differential Equations, Oxford Science Publications, The Clarendon Press, Oxford University Press, New York, 1995.

[18] B. D. Sleeman, Integral representations for solutions of Heun's equation, Proc. Cambridge Philos. Soc. 65 (1969), 447-459.

S. Belmehdi: UFR de Mathematiques Pures et Appliquées, Université des Sciences et Technologies de Lille, 59655 Villeneuve d'Ascq Cedex, France

E-mail address: belmehdi@athena.univ-lille1.fr

J.-P. Chehab: UFR de Mathematiques Pures et Appliquées, Université des Sciences et Technologies de Lille, 59655 Villeneuve d'Ascq Cedex, France

E-mail address: chehab@athena.univ-lille1.fr 


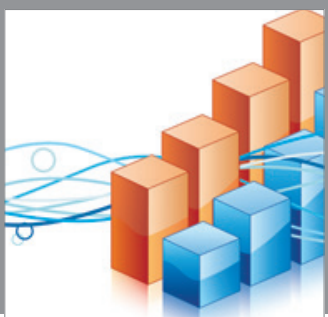

Advances in

Operations Research

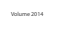

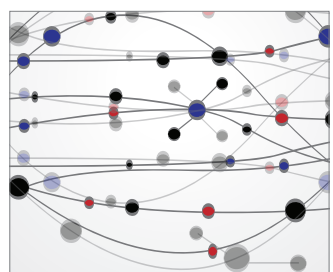

\section{The Scientific} World Journal
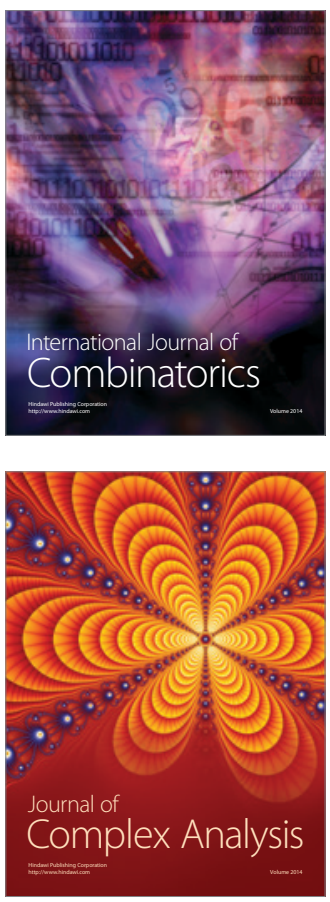

International Journal of

Mathematics and

Mathematical

Sciences
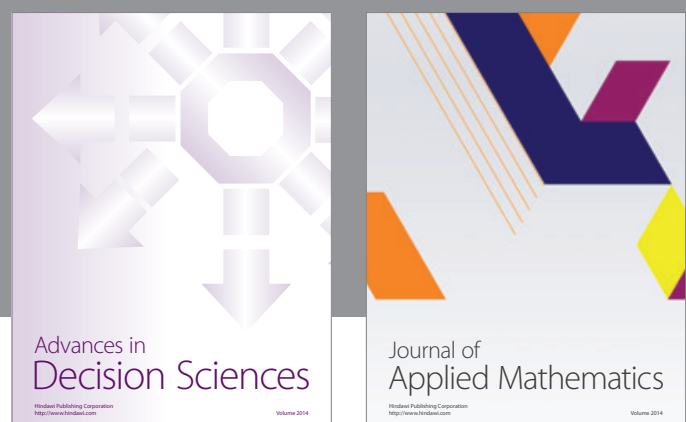

Journal of

Applied Mathematics
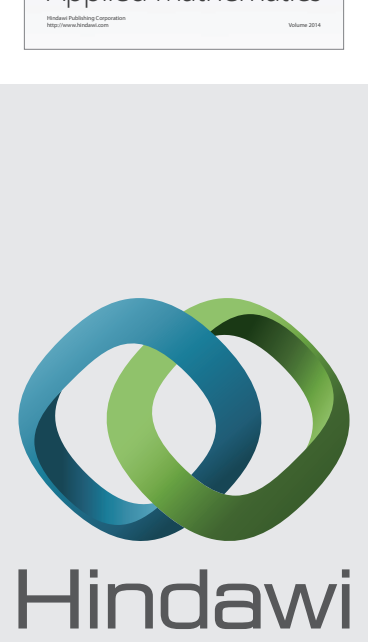

Submit your manuscripts at http://www.hindawi.com
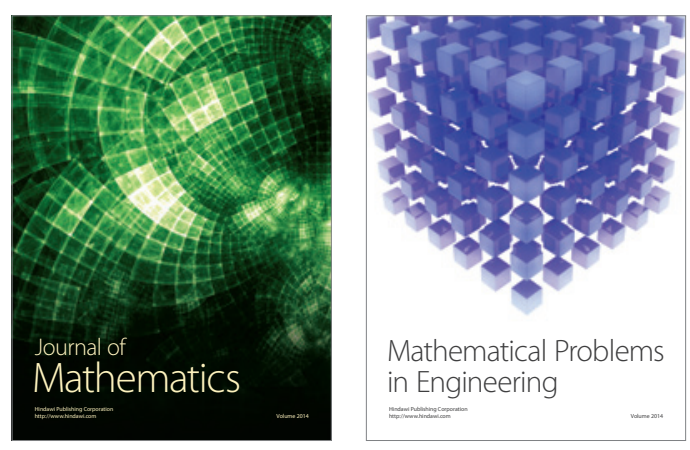

Mathematical Problems in Engineering
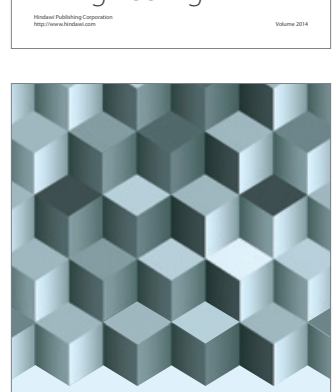

Journal of

Function Spaces
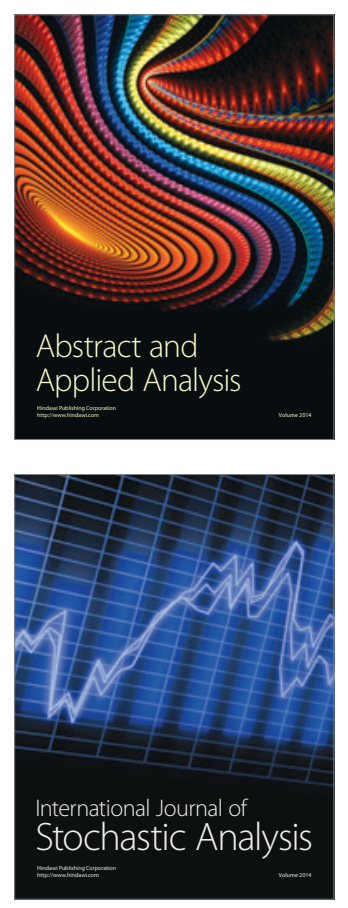

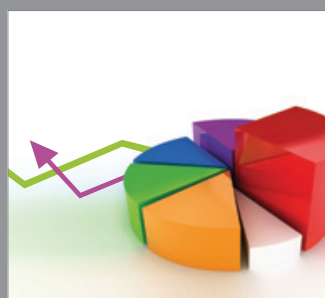

ournal of

Probability and Statistics

Promensencen
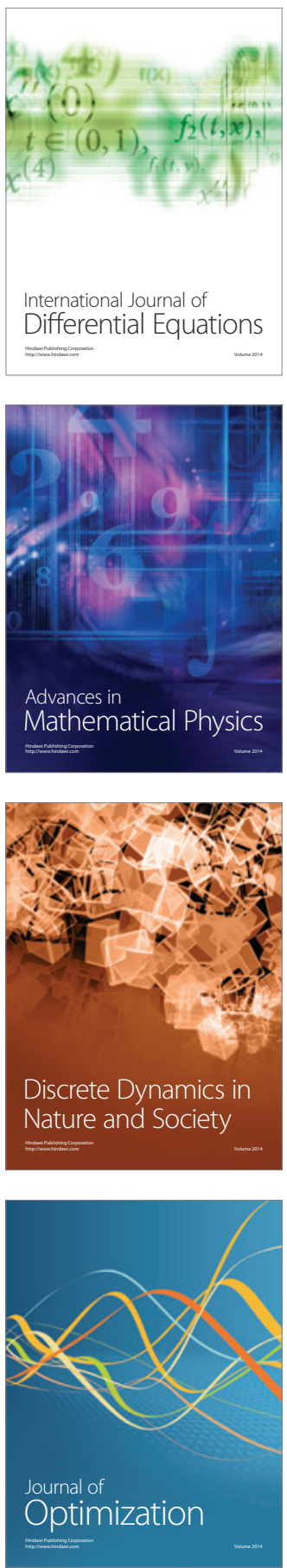